\title{
Graphical Statics Redux
}

\section{Dr. Sarah Baxter, University of St. Thomas}

Dr. Baxter is a Professor of Mechanical Engineering in the School of Engineering at the University of St. Thomas in St. Paul, MN. She received her PhD in Applied Mathematics from the University of Virginia School of Engineering and Applied Science.

\section{Dr. Bethany Fralick, University of South Carolina, Aiken}

Dr. Fralick is an Assistant Professor of Engineering in the Department of Mathematical Sciences at the University of South Carolina Aiken in Aiken, SC. She received her Ph.D. in Mechanical Engineering from the University of South Carolina College of Engineering and Computing. 


\title{
Graphical Statics Redux
}

\begin{abstract}
Active learning is defined in contrast to learning by exposition or lecture. More specifically it usually refers to any instructional activity that engages the student in learning, perhaps rather than in being taught. There are two fundamental challenges to embedding active learning in a Statics classroom. The first is coming up with the format for an activity that is appropriate for the class and learning objectives and the second is in identifying content that can be effectively cast in this format throughout the course.
\end{abstract}

In this work, Graphical Statics is suggested as an activity that addresses these challenges. Graphical Statics refers to methods that are based on the graphical method of adding vectors e.g., force and position, to solve two-dimensional Statics problems. The aims of the paper are as follows (1) to discuss how Graphical Statics fits into the context and criteria of active learning based on definitions and existing research in the literature; (2) to review the fundamental techniques of graphical statics, including force triangles and funicular polygons; (3) to outline a series/progression of concepts, in the order they appear in Statics curriculum, that can be solved using graphical techniques; and (4) to explore how the specific effects of these activities can be assessed.

\section{Active Learning}

Active learning is most generally defined as instructional techniques that emphasize activities other than traditional lectures. It was brought to popular attention in 1991 in the Bonwell and Eison $^{1}$ article for an Association for the Study of Higher Education (ASHE) report. It specifically refers to any instructional activity that engages the student in learning by doing as opposed to watching and listening. Students are required to engage in an activity, for example, writing, reading, discussing or problem solving that requires that they think about what they are doing how they are doing it and potentially why they are doing it. These activities are active learning in the sense that they promote more independent thinking; where independence is defined with respect to the instructor's direct guidance. If successful, these activities can switch the balance to learning from teaching and foster a more long-term internalization of the content. Since many engineering students are better at deriving than memorizing, active learning can help them make the connections between concepts, in addition, and as an aide to, acquiring and retaining knowledge. Active learning has been well researched in the educational literature. Most notably, the papers by Felder and Brent, ${ }^{2,3}$ focusing on collaborative-cooperative activities, have helped confirm the effectiveness of these approaches by documenting higher academic achievement, better high-level reasoning and critical thinking skills for students in STEM. Here, collaborative activities are broadly defined as instructional activities where students work together toward a common goal and cooperative learning refers to collaborative learning where students pursue a common goal but are assessed individually.

In "Does Active Learning Work: A Review of the Research", the authors conclude that there is strong evidence that engineering students remember more content if brief collaborative/cooperative activities are introduced in the lecture. They note that evidence also opposes traditional beliefs that individual work and competition best promote achievement. 
Freeman et al. ${ }^{5}$ meta-analyzed 225 studies that reported data on examination scores and/or failure rates when comparing STEM student performance under traditional lecturing versus active learning. The included studies covered a wide array of active learning activities, including cooperative group activities in class, but also in class worksheets, clickers, problem-based learning, and studio classrooms. Active learning activities ranged from 10-100\% of class time. Their results showed that on average student performance increased by just under half a standard deviation with active learning. With respect to failure rates, they found that students in traditional lecture classes were 1.5 times more likely to fail than students in classes with active learning. While there are caveats to the results presented in the study; e.g. active learning was not mandated, the instructors in these studies included it voluntarily and metrics of success and failure were not uniformly identical; these results strongly support including active learning activities in STEM classrooms.

\section{Active Learning In Statics}

There are two fundamental challenges to imbedding active learning in the Statics classroom. The first is coming up with the format for an activity that is appropriate for the class and learning objectives; the second is developing content that can be effectively cast in this format throughout

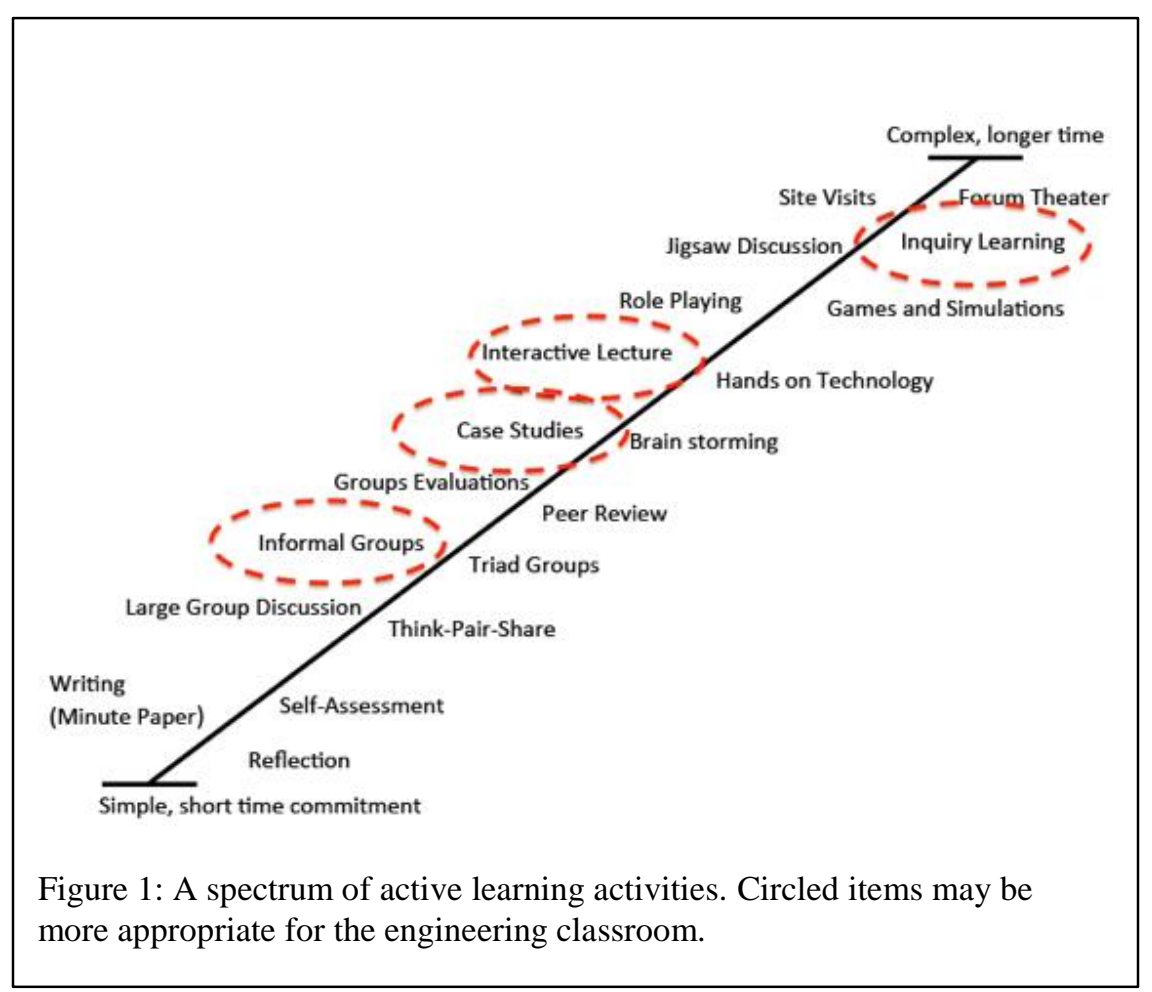
Forum Theater proposes using a stage set to depict a problem and have students act out the solution. Others are only useful strategies if the students could reasonably be expected to have some prior basic knowledge or opinions on the content; e.g. Discussions and/or Brainstorming, where the instructor introduces a topic or problem and then asks for student input and discussion.

A select few, highlighted on the figure, are compelling to engineering educators, for example, Informal Groups, Case Studies and Inquiry Learning. For the Statics classroom, informal groups 
could be as straightforward as groups of students working together on problems; simple case studies can provide more innovative problems and help motivate the need for a static analysis in engineering applications. Inquiry learning which the CRLT defines as students using an investigative process to discover engineering concepts on their own, is more challenging to employ, but could be used effectively for specific concepts (the role of reaction forces in equilibrium, static indeterminacy). In general, these activities are limited by the simplicity of the problems used to illustrate the concepts of Statics and their, in general, right or wrong answer.

The most obvious active learning activity is to pull homework and problem solving into the classroom. Students can work independently or in small groups, fostering their roles both as students and teachers, in a collaborative model. Additionally, some of the frustration, that often results when students get stumped, can be lessened by the presence of the instructor available to help. Students begin to see homework as a method of learning, through practice, rather than just a grade in the class. While this is likely an effective approach, it lacks some of the innovation and imagination that are present in many of the proposed active learning activities.

\section{Graphical Statics}

Graphical Statics, variously Graphic Statics and Graphical Analysis, is based on geometric construction. The most fundamental application provides the solution to adding vectors; briefly, when vectors are drawn to scale and arranged sequentially tail to head, their sum can be determined by measurements of the drawing. The foundations of graphical methods for engineering problems can be traced back to Leonardo da Vinci and Galileo Galilei. German engineer Karl Culmann, however, is generally considered the father of graphical statics. ${ }^{6}$ Early works formalized these methods for engineering, ${ }^{7,8,9}$ and the topic is of on-going interest in teaching structural design to architecture students, ${ }^{10,11}$ in emphasizing the significance of form in design to civil engineering students ${ }^{12,13,14}$ and was recently the focus of a doctoral dissertation exploring methods for generating thrust diagrams in statically indeterminate structure. ${ }^{15}$ Today, the techniques are often introduced in lectures on adding vectors, but rarely used in follow up problem solving.

\section{Fundamental Techniques - Force Polygons}

The basis of graphical statics is the idea of a force polygon. Each force vector is represented by an arrow, with direction, both line of action (an angle $\theta$ ), and sense (e.g. up or down) and magnitude (length of the arrow shaft). A series of forces, drawn to scale (magnitude of the force) and direction $(\theta)$, can be added together by placing the tail of each vector at the head of the previous one in the series. The sum of the forces is then the vector that runs from the tail of the first to the head of the last. This resultant vector closes the loop and

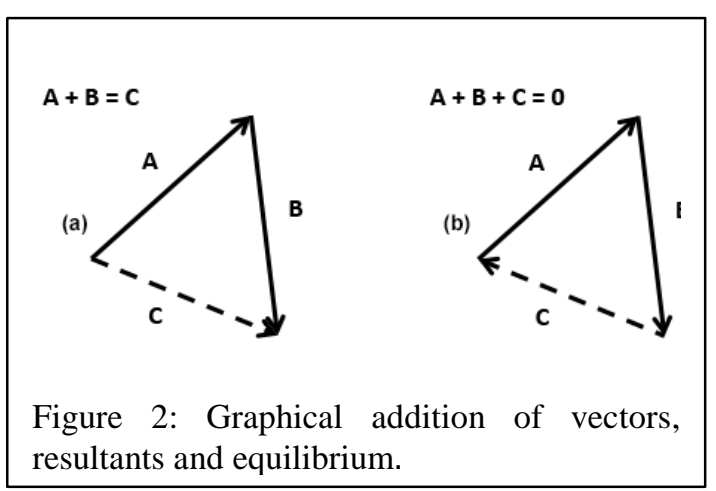
creates a force polygon, fig.2a. If the net force is zero, then the forces themselves close the loop, as the resultant is zero, fig. $2 b$ and there is force equilibrium. In simple statics problems, three forces in equilibrium form a force triangle. With more forces, equilibrium produces a force 
polygon. For systems of concurrent forces, the sole condition for equilibrium is that the sum of the forces is zero. For non-concurrent forces, moment equilibrium is required.

\section{Fundamental Techniques - Funicular or Equilibrium Polygons}

Funicular polygons, or equilibrium polygons, used in combination with force polygons, take into account the location of the forces. For non-concurrent forces, they can provide the additional information as to how to satisfy moment equilibrium and determine reaction forces. These geometric constructions mimic the response of a hanging cable to discrete loads; they are rarely used in Statics classes, but frequently used as a basis for architectural design.

While funicular polygons can be easily constructed by following a step-by-step procedure, their underlying base, why they work, is more sophisticated. To illustrate both the process and some of the base, two examples of their use are provided: determining the location of an equivalent resultant force and determining the reaction forces on a simply supported beam.

To determine an equivalent system of non-concurrent forces applied to a beam, initially a force polygon is used to determine the magnitude and direction of the resultant force due to a particular loading, fig.3a; a funicular polygon is then used to determine the location where the resultant force acts.

The process starts with an arbitrary pole point, $o$, selected to one side of the force polygon. Rays are drawn from the pole point to each of the heads or tails of the forces in the polygon, $o a, o b$, $o c, o d$ in fig. $3 \mathrm{~b}$. The rays are components of each of the forces that they bound, a non-orthogonal decomposition. Therefore each force can be replaced by the combination of those rays. Based on the parallelogram law, the construction can be illustrated by joining the two tails of each component force and observing that the line of action of the resultant force runs through this intersection (fig.4). The ray $o a$ is, therefore, applied to any point along the line of action of the first force, $F_{1}$, and $o b$, is applied at that same point.

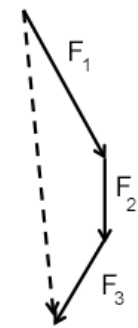

(a)

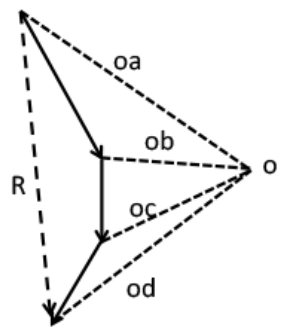

(b)

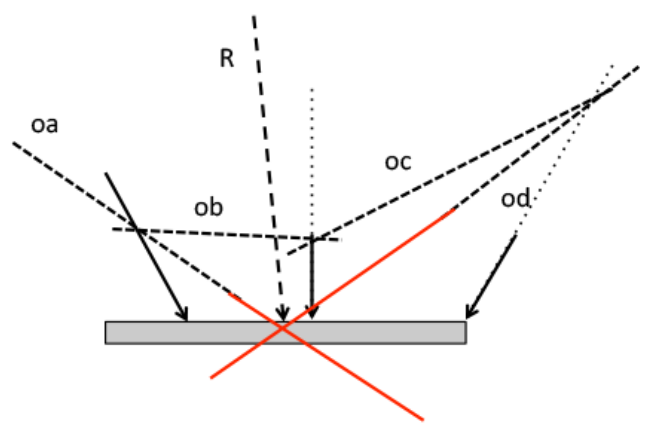

(c)

Figure 3: (a) The resultant force, $\mathrm{R}$, magnitude and direction is determined by constructing a force polygon, adding the forces together; (b) rays are drawn from the pole point to the tail and head of each force. These rays form a triangle with individual forces and represent a non-orthogonal decomposition of each force. (c) The funicular polygon is constructed using the components of each force. The intersection of the extensions of the lines of action of the initial and final components defines a point through which the line of action of the resultant must pass. 


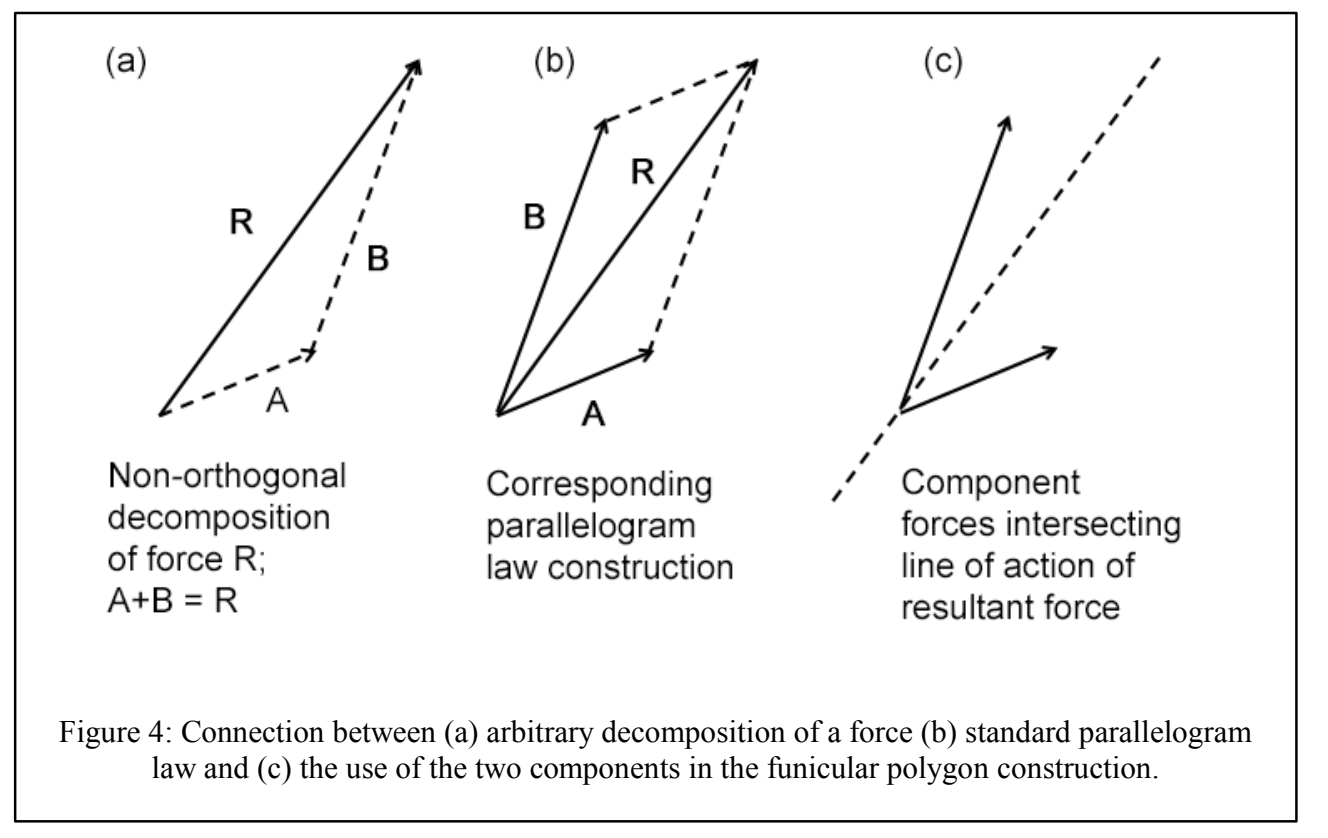

This corresponds to a parallelogram law description of the decomposition of a force. At the point where $o b$ intersects the line of action of the second force, the second component of the next force, $o c$, is applied; the process continues through the third force and its components, fig. $3 \mathrm{c}$.

Consider the force triangle, $o a+o b=F_{1}$ in fig. $3 \mathrm{~b} ; o b$ acts in the right to left direction. When the force triangle $o b+o c=F_{2}$ is considered, $o c$ is added, intersecting $o b$ and $F_{2}$. The process continues with the addition of $o d$, intersecting $o c$ and the line of action of $F_{3}$. The funicular polygon is based on the shape bounded by oa-ob-oc-od, with each ray beginning and terminating at its intersection with the line of action of a force.

The use of Graphical Statics to solve for reaction forces in a simple beam problem is shown in fig. 5. A beam is loaded with parallel forces of known magnitude and location (top left). The force triangle is, in this case just a line, known as the load line; it is drawn to scale. The resultant force is a line of exactly the same length overlapping the load line, but running in the opposite direction. This resultant force represents the sum of the two reaction forces.

A pole point, $o$, is again placed at an arbitrary position and rays drawn from the pole to each of the head and tail ends of the forces in the load line. These lines, with the same slope, are duplicated across lines marking the spatial positions corresponding to each applied force. Each of these lines begins and ends at the spatial position line. A final ray is drawn (solid red) from the first position to the last forming the funicular polygon. This line is called the closing string. If the line is translated, preserving its slope, onto the load line/pole drawing (bottom right) it bisects the resultant force into the magnitudes of the reaction forces. 

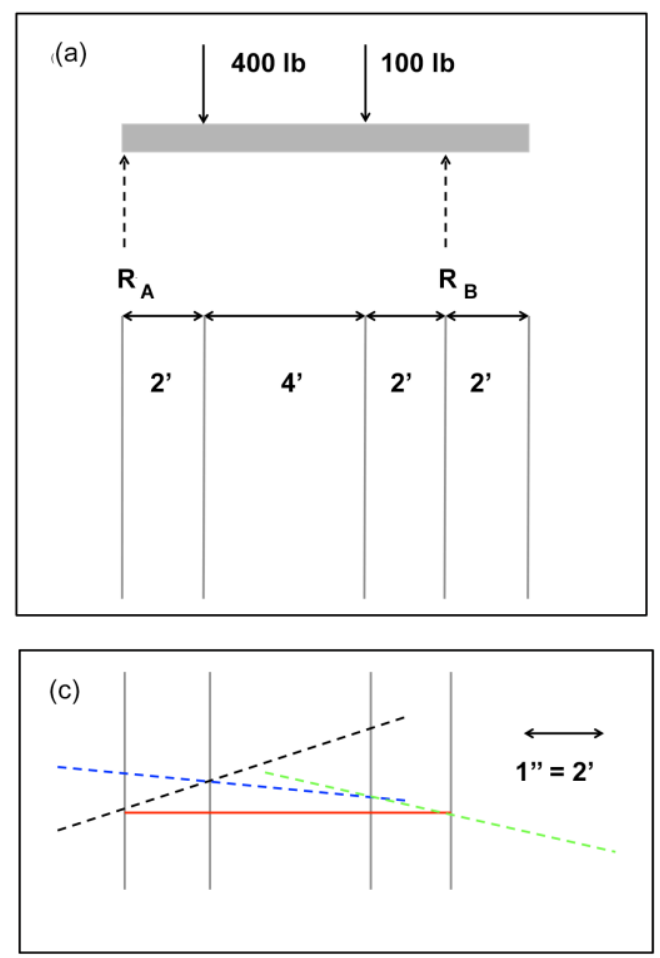

(b)
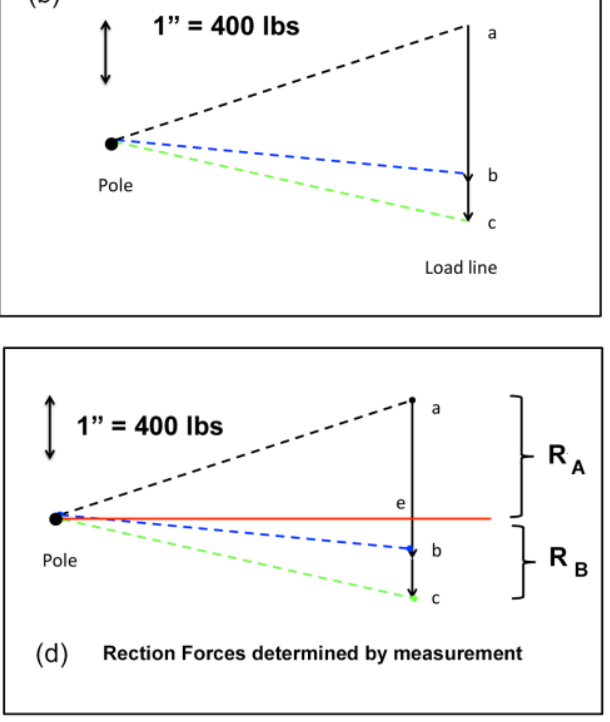

Figure 5: Reaction Forces on a Beam. (a) Free body diagram of the beam and spatial placement where forces are applied along the length (b) Force triangle is a (load) line, resultant force lies along same path, opposite direction. Rays connecting load line to pole point are force triangles of each applied force (c) Rays are used to construct a funicular polygon across spatial lines. The line from the intersection of the beginning ray to the end of the final ray closes the funicular polygon. When the red line is duplicated on the figure in $(\mathrm{d})$, running through the pole point, it bisects the load line into the lengths/magnitudes of the two reaction forces.

\section{Graphical Statics as Active Learning}

The success of specific active learning activities will be different for each student depending on their individual learning styles. ${ }^{3}$ An advantage of Graphical Statics is that it taps into a number of these styles. As examples, drawings and schematics connect well with sensing and visual learners; measurement is a hands-on activity, appealing to specifically active learners; and the process of solving a problem first, using Graphical Statics and second, using the equations of equilibrium connects well with sequential learning through practice. When both the graphical and mathematical solution are used to solve the same problem, sometimes the math becomes more visual.

Graphical Statics also functions well in the role of a collaborative or cooperative learning activity. Small groups of students are asked to work together; half of the team works to solve the problem analytically, and the other half to solve the problem using graphical methods. For a second problem, the half-team assignments are reversed. Solutions can be compared and 
contrasted. Competition exists in the comparison of results and teamwork is evident in working toward the same goal. In the authors' classrooms, these activities have not only promoted a collaborative environment and fostered teamwork, but resulted in student discussions as to (1) whether the math is 'right' and the drawings are 'wrong' and (2) is the math 'right', but the drawing more 'realistic' if you were designing something. Students were more willing to re-draw a solution, than re-run the numbers in their calculator. Additional discussions arose around more practical issues, such as the observation that larger scale drawings had less error, as compared to the analytic solutions, and if the pole point is placed closer to the force triangle, the slopes of the rays are more distinct (from each other) and so easier to draw.

\section{Graphical Statics Within the Curriculum}

Part of the advantage that Graphical Statics active learning activities may offer is their ability to be applied throughout the curriculum. However, many of the constructions and applications of graphical statics available in today's architecture and civil engineering texts are rooted in structural engineering design and are presented for students with existing, related knowledge in the area. In order to encourage the use of these techniques in a sophomore level Statics class, in what follows an outline of specific problem/concepts that are well suited to this level.

In companion work, also presented at this conference, sample problems with solutions, corresponding for each topic/concept presented in this outline, have been assembled into a minitextbook to facilitate the use of Graphical Statics. This textbook will be available at a poster presentation illustrating key components of the graphical approach, as well as from the authors by email (address will be included in final version of the paper). The textbook includes both graphical and mathematical solutions for each problem.

\section{Force Resultants}

This is the area where it is easiest to find a variety of problems that work well with Graphical Statics; simple resultants, a criterion for establishing equilibrium; decomposition along nonorthogonal axes.

a. Calculate the resultant force given the magnitudes and directions of two forces.

$b$. Find the magnitude and direction of a force that, when combined with a force of known magnitude and direction, produces a specified resultant.

c. Given the magnitude, but no directions, of two forces, and given the magnitude of the resultant, determine the directions of the two forces.

d. Decompose the given force along two non-orthogonal axes.

\section{Two-Dimensional Equilibrium of Concurrent Forces:}

This is essentially the problem associated with the laboratory exercise based on force tables. In each case, one force is completely specified, magnitude and direction. Most of the "book problems" are cases where the magnitudes of the forces are unknown, not the directions. When the directions are unknown, the result is a system of non-linear equations. The power of graphical statics is that it allows students to solve these systems of non-linear equations graphically. It introduces the notion that a force with a magnitude, but no specified direction, 
can be represented by the points along as circle with radius equal to the magnitude. This also strengthens the concept of a line of action.

a. Given the directions of the two other forces, determine their magnitudes.

$b$. Given the magnitudes of the two other forces, determine their directions.

c. Given the direction of one and the magnitude of the other force, determine their magnitude and direction, respectively.

\section{Beam Reaction Forces}

Beams make an excellent platform for reaction forces, equivalent loadings and distributed loads.

a. Determine the reaction forces for a beam with vertical loads and end supports.

b. Determine the reaction forces for an overhanging beam with vertical loads.

c. Determine an equivalent load; beam with not necessarily vertical loads.

\section{Centroids}

This method mimics the experiment where a weighted string is attached to the edge of an object of uniform mass/density; the line of the string is recorded. This is repeated with the string at a different location. Where the lines cross is the centroid. In Graphical Statics, the problem is solved several times. Each solution assumes gravity acts in a different direction.

\section{Simple Trusses}

Texts on architecture make significant use of graphical statics for trusses. However, it is mostly useful for complex trusses and in those cases the method requires a lot of time/practice to learn. The sample textbook does not include examples for trusses because of this time requirement.

\section{Shear and Moment diagrams}

Not all Statics classes include Shear and Moment diagrams. One example of a moment diagram is included in the sample problems because it corresponds well to graphical methods which are already used in teaching students to construct these diagrams.

\section{Additional Topics}

Discussions with students and other faculty suggested a number of other topics/skills that are reinforced by working some problems using Graphical Statics.

a. Scaled drawings and unit conversion.

b. Measurements; accuracy and error.

c. Encouraging sketching and use of drawing in design and analysis.

d. As a means of determining if the answer make sense.

e. Application for animations and scaled drawings in Solid Works. 


\section{Assessment}

Active learning is an established, well-assessed approach. Solving Statics problems in-class, cooperatively as well as independently, easily fits into the general category of active learning, There is evidence that even small time commitments to active learning have a positive effects on student achievement. From this perspective, no specific assessment of the effectiveness of adding modules based on Graphical Statics is necessary. The challenge for the Statics classroom is more on finding valid active learning activities than on justifying active learning.

However, the authors think that graphical techniques add value beyond just functioning as active learning exercises. A more focused assessment of the specific skills and insight linked to these activities is a current and future goal of the project. It has been particularly challenging given that (1) the proposed activities, even with enthusiastic support, do not represent a significant time component of the class and (2) the added value may be in higher-level outcomes, e.g. visualization of concepts, solving open ended problems; which are difficult to assess. ${ }^{4}$ Thus, the Statics Concept Exam ${ }^{16}$, a highly visual test, may be good way to assess visualization but is affected by other components of the class such as other active learning activities, demonstrations, laboratory experiences, as well as the form and formats of traditional lectures.

One anticipated educational benefit of graphical analysis, that may allow focused assessment, is the potential to enhance students' ability to answer questions of "does this answer make sense?" Engineers frequently sketch load lines, forces and geometry to help answer these types of question. Future work, therefore, will include the development of a short quiz that asks for some "does this make sense" analysis. Example questions such as below would be those that could be answered qualitatively by drawing/sketching, but without calculators.

(1) Given the magnitudes of two forces, but not their directions, what is the maximum resultant force, the minimum?

(2) Given three forces with directions, all measured from the positive $x$-axis, of $45^{\circ}, 20^{\circ}$ and $120^{\circ}$, is it likely that the system is in equilibrium?

(3) What is the impact of larger or smaller angles shown here?

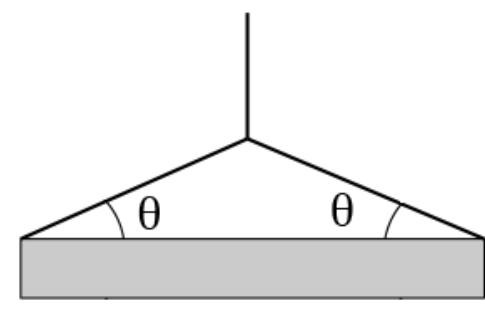

(4) Compare the mechanical advantage of pliers with different length of blades/handles?

(5) Is the order of magnitude of this solution correct? 


\section{Conclusions}

In previous work ${ }^{17}$, the authors proposed enhancing the Graphical Statics component in Statics classes. The hypothesis posed was that adding additional Graphical Statics components would help build visualization skills, encourage drawing as a conceptual aid, and help reinforce the concepts.

The response, during the presentation of this previous work, acknowledged the potential for these outcomes, but the audience was more focused on questions about specific Graphical Statics problems that could be directly inserted into the class as active learning exercises. From a practical perspective, it was suggested that a framework and progression of Graphical Statics examples, that could become a consistent active component of the class, would be useful and motivate the use of this approach. This became the focus of the current work.

As content for Graphical Statics was identified and developed, it was apparent that graphical solutions also have the potential to mimic the design sequence; constructing the drawing represents design; measurements represent an experiment; and comparisons with mathematical solutions, represent an analysis phase. This brings in more of the imagination and innovation that is characteristic of the best of active learning. Finally, with a consistent thread of activities, it is possible to include a graphical statics component in Statics classes is such a way that it supports and reinforces the existing content but does not unduly burden an already crowded curriculum.

Future work will focus on identifying the unique contributions of Graphical Statics within the active learning framework and developing ways to assess student growth and achievement in these areas.

\section{References}

[1] Bonwell, C.C. and Eison, J.A. “Active Learning: Creating Excitement in the Classroom, ASHE-ERIC Higher Education Report No. 1. Washington D.C.: The George Washington University School of Education and Human Development, 1991.

[2] Felder, R. and Brent, R. "Cooperative Learning in Technical Courses: Procedures, Pitfalls and Payoffs" ERIC Document Reproduction Service, ED 377038, 1994.

[3] Felder, R. and Brent, R. "Learning by Doing" Chem. Engr. Education 37(4), 282-283, 2003.

[4] Prince, M. "Does Active Learning Work? A Review of the Research" J. Engr. Education, 93(3) 223-231, 2004

[5] Freeman, S., Eddy, S.L., McDonough, M., Smith, M.K., Okoroafor, N. Jordt, H and Wenderroth, M.P. "Active learning increases student performance in science, engineering and mathematics" PNAS, 111(23), 8410-8415, 2014.

[6] Culmann. Karl, "Die graphisehe Statik" Zürich: Verlag van Meyer \& Zeller, 1864/1866.

[7] Wolfe, W. S. Graphical Analysis a Text Book on Graphic Statics McGraw-Hill Book Co., Inc. NY., 1921.

[8] Malcolm, C.W. "Text Book on Graphic Statics" Thesis for Degree of Civil Engineer in Civil Engineering, College of Engineering, University of Illinois, Presented June 1906.

[9] Cathcart, W.L. and Chafee, J.I. " A Short Course in Graphic Statics for Students of Mechanical Engineering" D. Van Nostand Co., N.Y. (BiblioBazaar Reproductions, LLC) 1911

[10] Dermody, R. J. "Embracing the Past: Using Historical structures to Teach Engineering Fundamentals" ASEE Annual Conference Proceedings, 2012.

[11] Dermody, R.J. "Get the Form Right!!"In Proceedings of the ASEE Annual Conference Proceedings, 2010.

[12] Zalewski, W. and Allen, E. Shaping Structures: Statics Wiley and Sons, Inc., 1998. 
[13] Gerhardt, R., Kurrer, K.-E., Pichler, G. "The methods of graphical statics and their relation to the structural form" Proceedings of the First International Congress on Construction History, Madrid, 20th-24th January 2003, ed. S. Huerta, Madrid: I. Juan de Herrera, SEdHC, ETSAM: A. E. Benvenuto, COAM: F. Dragados, 2003.

[14] Allen, E., Zalewski, W., Foxe, D.M., Anderson, J., Hriczo, J.K., Ramage, M.H., Ochsendorf, J.A., Block, P. and Iano,J . Form and Forces: Designing Efficient, Expressive Structures Wiley and Sons, Inc. 2010.

[15] Liem, Yannick "Graphic statics in funicular design calculating force equilibrium through complementary energy", Graduate Thesis TU Delft, Faculty of Architecture.

[16] Baxter, S. C., Johnson, A., and Fralick, B. S. "Revisiting Graphical Statics” In Proceedings of the ASEE Annual Conference and Exposition, Seattle, Washington. 10.18260/p.24681, 2015 